\title{
Detection of Cervical Cancer Based on Learning Vector Quantization and Wavelet Transform
}

\author{
Latifah Listyalina*1 $^{*}$, Dhimas Arief Dharmawan ${ }^{2}$ \\ ${ }^{1}$ Department of Electrical Engineering, Faculty of Scince and Technology, Universitas Respati Yogyakarta \\ J1. Laksda Adisucipto KM 6,3 Depok Sleman Yogyakarta 55281, Telp (0274) 488781 \\ ${ }^{2}$ Department of Electrical Engineering, Faculty of Engineering, Universitas Muhammadiyah Yogyakarta \\ Jalan Brawijaya, Geblangan, Tamantirto, Kasihan, Bantul 55183, Telp (0274) 387656 \\ *Corresponding author, e-mail: listyalina@respati.ac.id
}

\begin{abstract}
Cervical cancer has became the common women disease in the world. Mostly, cervical cancer has been already known lately, because it is very difficult to detect this in early stage. In this work, a computer based software using Learning Vector Quantization (LVQ) has been designed as the early cervical cancer detection aid tool. There are six methods before the detection is performed, namely preprocessing, contrast stretching, median filtering, morphology operation, image segmentation, and Wavelet Transform based feature extraction. In this work, 73 cervical cell images that consist of 50 normal images and 23 cancer images are used. 35 normal images and 14 cancer images are used to train the LVQ. Then, 23 normal images and 9 cancer images are used in the testing process. Our results show $88.89 \%$ cancer image can be detected correctly (sensitivity), $100 \%$ normal image can be detected correctly (specificity), and 95.83\% for overall detection (accuracy).
\end{abstract}

Keywords: Cervical Cancer, Learning Vector Quantization, Wavelet Transform

\section{Introduction}

A Cervical cancer is included in one type of cancer ferocious attacks on women. According to WHO data, the number of cervical cancer sufferers in Indonesia is very high. Every year, no less than 15,000 cases of cervical cancer happened in Indonesia. This makes WHO put Indonesia as a country with cervical cancer sufferers most in the world [1].

Most cervical cancer cases are caused by viruses, namely Human Papilloma Virus (HPV). So far, there are more than 150 known types of HPV viruses. Between known types of viruses, HPV types 16 and 18 is the most widely known cause onset of cervical cancer. Spread of HPV can occur due to skin contact. In most cases, spread HPV is more common due to sexual relations [1].

Prevention of this disease can be done by detection early through a pap smear test. Output from pap smear test is the image of cervical cells.
From the image of cervical cells produced, cervical cell can be determined into categories normal or cancer. Detection of cervical cells as normal cells or cancer cells can be done in two ways, viz through visual observation, and with help computer. In general, detection of cervical cancer is done by a doctor through visual observation.

Detection through visual observation by a doctor has some disadvantages, one of which is. To overcome this, the existence of a software based computer for the detection of cervical cancer based on cervical cells very expected presence. There is software computer-based cervical cancer detection is expected able to extract and process cervical cancer characteristics more effective than visual observation, so the detection results obtained have a level high accuracy.

Several studies regarding the detection of cervical cancer some of which are performed automatically by [1] and [2]. Reference [2] uses the area and circumference features of the cell nucleus cervix as the method. This research resulted 
accuracy of $82 \%$. Reference [1] uses area features, circumference, shape and roundness of the cervical cell nucleus and LVQ as the method. This research yields accuracy better by $93.3 \%$. In that study, the authors states that the feature extraction method is needed other cervical cells that are able to increase the accuracy of software. To increase the level of accuracy like This suggestion [1], in this study was designed software for cervical cell classification efforts from images Pap smears using Learning neural networks Vector Quantization (LVQ). This method is known to be more effective in terms of sensitivity, specificity, accuracy, and computational burden compared to the backpropagation method [3].

\section{Methodology}

The process of detection of cervical cancer consists of eight pieces stages, namely preprocessing, contrast stretching, median filters, morphological operations, segmentation, feature extraction with Wavelet Transform, LVQ network training, and detection of cervical cancer with LVQ. The first stage of the method of detection of cervical cancer is preprocessing. Preprocessing is divided into two stages, namely cropping and grayscalling. Cropping phase aims to uniform the size of the image of cervical cancer to be measuring $150 \times 150$ pixels. The second stage of preprocessing is grayscalling. Grayscalling aims to change the image cervical cancer, from RGB images to grayscale images. Imagery RGB is a type of image that presents deep colors component form R (red), G (green), and B (blue). Every color components use 8 bits (values range between 0 to 255). Color representation of RGB images shown in Figure 1.

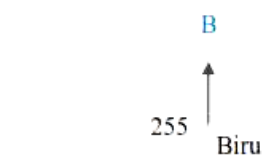

Magenta

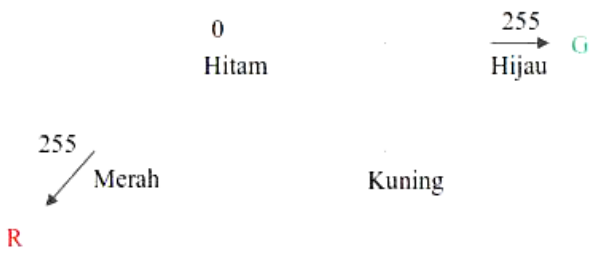

Fig. 1. RGB color in three dimensional space [4]
In addition to RGB images, there are also grayscale images or images gray scale. this type of image handles color gradations black and white, which of course produces a gray effect.

In this type of image, colors are expressed in intensity between 0 and 255. A value of 0 represents black and value 255 states white. Transform RGB image into Grayscale images are carried out according to the equation below [4]. I = $0.2989 \mathrm{R}+0.5870 \mathrm{G}+0.1141 \mathrm{~B}$ (1) The next step is to stretch contrast. Contrast on an image can be defined as the distribution of bright pixels and dark in an image. Image with good contrast displays a wide range of pixel values. Histogram of image with good contrast shows the distribution of values pixels that are uniform, don't have a main peak, and also has no valleys.

Stretching contrast is one technique for improve image contrast. This technique works well in images that have a gaussian or close distribution gaussian. In this study, contrast stretching was calculated according to the following equation [5].

$$
o(i, j)=\frac{u(i, j)-c}{d-c}(L-1)
$$

With o (i, j) and $u(i, j)$ successive pixels after $d$ - c (L-1) and before transformation in coordinates $(i, j), c$ and $d$ respectively states the maximum and minimum pixel values of the image input, while $\mathrm{L}$ represents the maximum pixel value output image [5].

Median filter becomes the next step. Median filter is one type of non-linear filter. Median filter is one filter that is commonly used, specifically to handle salt and pepper noise. Median filter implemented through the three steps below [6]:

1. Determine the size of the median kernel filter to be used.

2. For each kernel, sort all pixel values, and Determine the median value of the set of pixel values.

3. The median value obtained is used for replaces the pixel value at the center of the kernel.

For the effort to localize the cervical cell nucleus, surgery is needed morphology. In this study, morphological operations were used is opening and closing operations. Second operation it is a second level operation, because it was formed by other morphological operations, namely erosion and dilation. Opening defined as erosion surgery followed by dilation operations using certain 
element structures. Conversely, closing is defined as a dilation operation followed by erosion operations using structural elements certain. The two operations can be formulated as follows [7].

$$
\begin{aligned}
& A \circ B=(A \ominus B) \oplus B \\
& A \cdot B=(A \oplus B) \oplus B
\end{aligned}
$$

With $\mathrm{A} \oplus \mathrm{B}$ and $\mathrm{A} \ominus \mathrm{B}$, respectively dilation and erosion surgery of $\mathrm{A}$ over $\mathrm{B}$. The segmentation phase is interpreted by the method Otsu floating. In the Otsu method, the value calculation a threshold in a segmentation process based on the clustering process. Image assumed has two groups of pixels according to their histogram, i.e. foreground and background. The optimum threshold value The result is a threshold value that is able to separate both groups of pixel values are as maximum as possible [8].

Wavelets are mathematical functions that cut up data into different frequency components, and then study each component with a resolution matched to its scale. They have advantages over traditional Fourier methods in analyzing physical situations where the signal contains discontinuities and sharp spikes. Wavelets were developed independently in the fields of mathematics, quantum physics, electrical engineering, and seismic geology. Interchanges between these fields during the last ten years have led to many new wavelet applications such as image compression, turbulence, human vision, radar, and earthquake prediction [11].

LVQ training becomes the next stage of detection cervical cancer. LVQ is a pattern classification method each unit of output represents a certain class [10]. The nomenclature used is:

$\begin{array}{ll}\mathrm{X} & =\text { vector trained } \\ \mathrm{T} & =\text { Class for trained vectors } \\ \mathrm{Wj} & =\text { Weight vector for the jth output unit } \\ \mathrm{Cj} & =\text { Class represented by the output unit to }\end{array}$

$\mathrm{Cj} \quad=$ Class represented by the output unit to $\mathrm{j}$. $\| \mathrm{x}$-wj $\|=$ Euclidian distance between the input vector and (vector weight) jth output unit.

The LVQ training process is carried out by including features training image into the LVQ artificial neural network. Process training is carried out to get the optimal weight value which is used to classify the test image. During training, the output unit is positioned in a way set the value of its weights through supervised training for estimate decision surfaces from Bayes classifiers.
As an initial step of the training, initialization needs to be done certain parameter values. The parameter value must be initialized are initial weight, maximum epoh, initial learning rate $(\alpha)$, minimum learning rate $(\alpha)$, changes in learning rate $(\alpha)$, and termination of the training process.

In this study, the parameter values mentioned above are initialized as follows: Weights are initialized by taking two image features consisting of one normal cervical cell image feature and one cancer cervical cell image feature; The maximum value of epoh is 100 epoch; The initial learning rate $(\alpha)$ value is 0.5 ; The value of the learning rate $(\alpha)$ of at least 10-6; Learning rate values $(\alpha)$ are updated with the rules $\alpha(\mathrm{t}+1)=0.5 \alpha(\mathrm{t})$; The termination condition is if the number of epoh has exceeded the maximum value of epoh, or the value of learning rate $(\alpha)$ has been less than the minimum learning rate value $(\alpha)$; The last process is detection of cervical cancer. The LVQ network mends the input vector by placing it in the same class as the output unit which has the weight vector closest to the input vector

\section{Result and Discussion}

In this paper, a method for detecting cervical cancer is applied to 73 images of cervical cells. The 73 pieces were then divided into two groups, namely the training image and the test image, each totaling five headlands and 23 pieces.

The 73 images have been processed before processing the feature extraction stage. Examples of the results of cervical cell image processing, starting from preprocessing to segmentation with the otsu method can be seen in Figure 2.

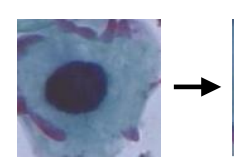

(a)

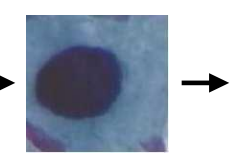

(b)
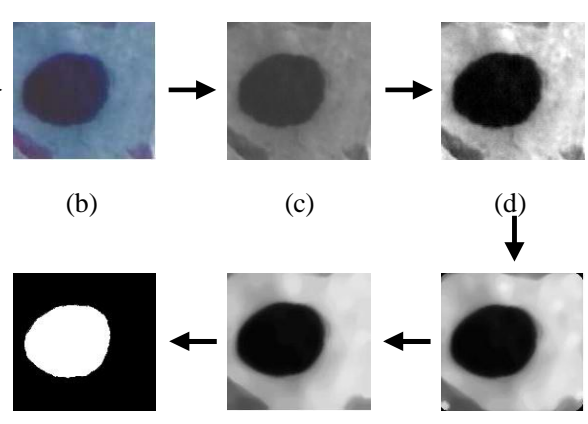

(g) (c)

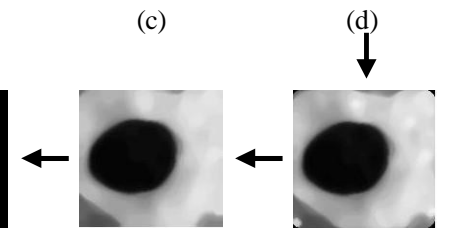

(f) (e)

Fig. 2. Image the results of cervical cell image processing, starting from preprocessing to segmentation with the Otsu method

In Figure 2, it appears that the purpose of the image processing series is to get the main 
information from the image of the cervical cell, namely the image of the nucleus of the cervical cell. Preprocessing which consists of cropping and grayscalling aims to uniform the size and format of the image. In addition, preprocessing also serves to reduce the computational burden due to smaller output image sizes and reduced output image channels.

Next, stretch contrast and median filters are used to improve image quality, as shown in Figure 2 (d) and (e).

The median filter applied is a multiple median filter with kernel sizes 3, 5, 7, and 9. The application of a set of wavelet transform values in that window. From this process an average $r$ value will be generated, and then arranged into feature vectors of each $1 \times \mathrm{r}$ image.

The LVQ training process is carried out by training the feature vectors of 35 normal cell image images and 14 cancer cell image images. Because the LVQ network is expected to have two output classes, the initial weight used is $2 \mathrm{xr}$, where the first row of the matrix comes from one of the input vectors of the cancer cell image, and the second row of the matrix comes from one of the normal cell image input vectors.

The training process is carried out with a number of conditions mentioned in the previous section.
This training process produces a $2 \times \mathrm{r}$ weight matrix, where the first row of the matrix shows the weight vector of the cancer cell image, and the second row of the matrix shows the weight vector of the normal cell image. Finally, the process of detecting cervical cancer is done by inserting feature vectors of 15 normal cell image images and 9 images of cancer cells.

The LVQ network detects the output of each input vector by placing it in the class that has the closest distance to the weight vector. The results of detection of cervical cancer by LVQ tissue can be seen in Table 1.

From Table 1 it can be seen that detection errors only occur in Test 8 images, where cancer images are detected as normal. That way, based on the detection results, the value of True positive (TP) is obtained, namely the number of images of cancer cells detected as cancer as much as 8. Furthermore, there is no False positive value (FP) that is the number of normal cell images detected as cancer. Whereas the value of True Negative (TN) and False Negative (FN), namely the number of norm cell images detected as normal, and the number of normal cell images detected as cancer respectively by 15 and 1 . Based on TP, FP, TN, and FN values above, we can calculate the values of sensitivity, specificity, and accuracy as follows:

TABLE I

DETECTION RESULTS

\begin{tabular}{cccccc}
\hline \hline Image & $\begin{array}{c}\text { Distance to } \\
\text { Cancer }\end{array}$ & $\begin{array}{c}\text { Distance to } \\
\text { Normal }\end{array}$ & $\begin{array}{c}\text { Detection } \\
\text { Result }\end{array}$ & Groundtruth & Remark \\
\hline Test 1 & 2.23 & 4.31 & Cancer & Cancer & Match \\
Test 2 & 2.24 & 5.78 & Cancer & Cancer & Match \\
Test 4 & 4.66 & 7.44 & Cancer & Cancer & Match \\
Test 5 & 1.96 & 5.76 & Cancer & Cancer & Match \\
Test 6 & 1.91 & 6.87 & Cancer & Cancer & Match \\
Test 7 & 5.29 & 5.70 & Cancer & Cancer & Match \\
Test 8 & 4.95 & 3.46 & Normal & Cancer & No Match \\
Test 9 & 3.88 & 9.58 & Cancer & Cancer & Match \\
Test 10 & 3.78 & 4.00 & Cancer & Cancer & Match \\
Test 11 & 5.41 & 0.88 & Normal & Normal & Match \\
Test 12 & 5.67 & 1.65 & Normal & Normal & Match \\
Test 13 & 6.79 & 0.97 & Normal & Normal & Match \\
Test 14 & 5.24 & 1.94 & Normal & Normal & Match \\
Test 15 & 6.06 & 1.41 & Normal & Normal & Match \\
Test 16 & 5.28 & 1.63 & Normal & Normal & Match \\
Test 17 & 4.41 & 1.79 & Normal & Normal & Match \\
Test 18 & 7.00 & 2.66 & Normal & Normal & Match \\
Test 19 & 5.53 & 0.88 & Normal & Normal & Match \\
Test 20 & 6.09 & 1.23 & Normal & Normal & Match \\
Test 21 & 4.71 & 1.85 & Normal & Normal & Match \\
Test 22 & 6.32 & 1.67 & Normal & Normal & Match \\
Test 23 & 5.65 & 1.78 & Normal & Normal & Match \\
Test 24 & 4.37 & 1.44 & Normal & Normal & Match \\
\hline \hline
\end{tabular}


Multiple median filters are caused by the amount of noise found in the image of cervical cells. With these two operations, cervical and background cells become easy to distinguish. In addition, the two operations make each light and dark area have an even pixel intensity.

Morphological operations are carried out by applying an opening operation followed by a closing operation. Morphological surgery functions to isolate the cervical cell nucleus, as shown by Figure 2 (f).

The isolated cervical cell nucleus makes the segmentation process by the Otsu method run more optimally. The use of the Otsu method is because the otsu method applies adaptive floating in the segmentation process. This makes each image has its own threshold value, which is more representative of the character of the image, rather than global floating.

Feature extraction of cervical cell images was performed using wavelet transform. Before wavelet transform is applied, the input image is first divided into a number of $\mathrm{n} \times \mathrm{n}$ windows. Each pixel in each window is calculated its wavelet transform value, then the average value is then calculated.

Referring to Table I, we could calculate the sensitivity, specificity, and accuracy values that are $88.89 \%, 100 \%$, and $95.83 \%$, respectively.

\section{Conclusion}

Software for Automatic Cervical Cancer Detection Based on Artificial Neural Network Learning Vector Quantization (LVQ) and wavelet transform from Pap Smear Image successfully designed through eight stages of the method, namely preprocessing, contrast stretching, median filter, morphological operation, segmentation, feature extraction with wavelet transform, LVQ network training, and detection of cervical cancer using the LVQ network. From the testing process, high sensitivity, specificity, and accuracy values were obtained, each at $88.89 \%, 100 \%$, and $95.83 \%$.

\section{References}

[1] E. M. Dewi, "Ekstraksi Fitur dan Klasifikasi Sel Serviks Dengan Metode Learning Vector Quantization (LVQ) Untuk Klasifikasi Dini Cancer Serviks," Universitas Airlangga, 2013.

[2] J. Norup, "Classification of Pap Smear Data by Transductive NeuroFuzzy Methods," University of Denmark, 2005.
[3] L. Listyalina, "Implementasi Learning Vector Quantization (LVQ) Untuk Klasifikasi Cancer Paru dari Citra Foto Rontgen," Universitas Airlangga, 2013.

[4] A. Kadir and A. Susanto, Pengolahan Citra. Yogyakarta: Penerbit ANDI, 2012.

[5] D. Putra, Pengolahan Citra Digital. Yogyakarta: Penerbit ANDI, 2009.

[6] R. C. Gonzalez and R. E. Woods, Digital Image Processing, 2nd ed. New Jersey: Prentice Hall, 2002.

[7] R. C. Gonzales, R. E. Woods, and S. L. Eddin, Digital Image Processing Using MATLAB, 2nd ed, 2nd ed. India: McGraw-Hill Education, 2009.

[8] N. Otsu, "No Title," IEEE Tras. Syst. Man Cybern, pp. 62-66, 1979.

[9] W. K. Pratt, Digital Image Processing, 4th ed. California: WILERINTERSCIENCE, 2007.

[10]L. V Fausett, Fundamental of Neural Network: Architectures, Algorithms, and Applications. Prentice-Hall, 1994.

[11]https://www.eecis.udel.edu/ amer/CISC651/IEEEwa velet.pdf

\section{Authors' information}

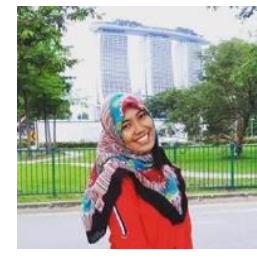

Latifah Listyalina is a lecturer at the Department of Electrical Engineering, Faculty of Science and Technology, Universitas Respati Yogyakarta. She received the B.Eng degree in Biomedical Engineering from Universitas Airlangga, Indonesia, in 2013 and the M.Eng. degree in Electrical Engineering from Universitas Gadjah Mada, Indonesia in 2016. Her research interests include biomedical signal and image processing, computer vision and pattern recognition.

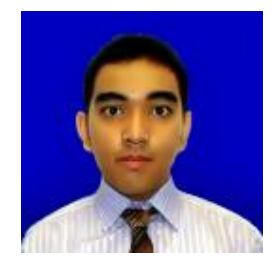

Dhimas Arief Dharmawan is a lecturer at the Department of Electrical Engineering, Faculty of Engineering, Universitas Muhammadiyah Yogyakarta. $\mathrm{He}$ received the B.Eng. degree in Electrical Engineering from Universitas Gadjah Mada, Yogyakarta, Indonesia in 2014. He is currently pursuing the Ph.D. degree in Electrical Engineering at Nanyang Technological University (NTU), Singapore. His research interests include image filtering and segmentation, machine learning, computer vision and pattern recognition. 